\title{
Concurrent SHORT syndrome and 3q duplication syndrome
}

\author{
Alexander M. Boaz"*, Salvatore A. Grasso', Michael J. DeRogatis, and Ellis N. Beesley ${ }^{1,2}$ \\ ${ }^{1}$ Department of Pediatrics, California Hospital Medical Center, Los Angeles, CA, USA \\ ${ }^{2}$ Department of Clinical Medicine, Ross University School of Medicine, Miramar, FL, USA
}

SHORT syndrome is an extremely rare congenital condition due to a chromosomal mutation of the PIK3R1 gene found at 5q13.1. SHORT is a mnemonic representing six manifestations of the syndrome: $(\mathrm{S})$ short stature, $(\mathrm{H})$ hyperextensibility of joints and/or inguinal hernia, $(\mathrm{O})$ ocular depression, $(\mathrm{R})$ Rieger anomaly, and $(\mathrm{T})$ teething delay. Other key aspects of this syndrome not found in the mnemonic include lipodystrophy, triangular face with dimpled chin (progeroid facies, commonly referred to as facial gestalt), hearing loss, vision loss, insulin resistance, and intrauterine growth restriction (IUGR). 3q duplication syndrome is rare syndrome that occurs due to a gain of function mutation found at 3q25.31-33 that presents with a wide array of manifestations including internal organ defects, genitourinary malformations, hand and foot deformities, and mental disability. We present a case of a 2 year and 3 month old male with SHORT syndrome and concurrent $3 q$ duplication syndrome. The patient presented at birth with many of the common manifestations of SHORT syndrome such as bossing of frontal bone of skull, triangular shaped face, lipodystrophy, micrognathia, sunken eyes, and thin, wrinkled skin (progeroid appearance). Additionally, he presented with findings associated with $3 q$ duplication syndrome such as cleft palate and cryptorchidism. Although there is no specific treatment for these conditions, pediatricians should focus on referring patients to various specialists in order to treat each individual manifestation.

Key words: Mutation, Syndrome, Chromosomes, Lipodystrophy, Fetal growth retardation.

\section{Introduction}

Patients with an extremely rare missense mutation in the phosphoinositide-3-kinase, regulatory subunit 1 (PIK3R1) gene present with an array of symptoms described as SHORT syndrome [1]. SHORT is a mnemonic representing six manifestations of the syndrome: short stature, hyperextensibility of joints, inguinal hernia, ocular depression, Rieger anomaly (malformation of the anterior chamber of the eyeball), and teething delay [2]. Other primary features of SHORT syndrome not included in the mnemonic are lipodystrophy, intrauterine growth restriction (IUGR), hearing loss, vision loss, insulin resistance, delayed growth, and facial gestalt. The facial gestalt of SHORT syndrome is comprised of a combination of slightly varying case-specific facial features including triangular face, prominent forehead, hypoplastic alae nasi, midface hypoplasia, micrognathia, large low-set ears, thin lip with downturned mouth, and progeroid face. The condition has a worldwide incidence of $<1 / 1,000,000$ and there have been less than 50 cases of SHORT syndrome reported in literature since the recognition and naming of the

Received: 29 June 2018, Revised: 5 November 2018, Accepted: 5 November 2018, Published: 30 June 2019

${ }^{*}$ Corresponding author: Alexander M. Boaz, B.S. (iD https://orcid.org/0000-0002-1585-9294

Department of Pediatrics, California Hospital Medical Center, 1401 S. Grand Ave., Los Angeles, CA 90015, USA.

Tel: +1-541-301-0765, E-mail: alexanderboaz@students.rossu.edu

Conflict of interest: The authors declare that they do not have any conflicts of interest.

(ㄷ) This is an open-access article distributed under the terms of the Creative Commons Attribution Non-Commercial License (http://creativecommons.org/licenses/by-nc/4.0/) which permits unrestricted non-commercial use, distribution, and reproduction in any medium, provided the original work is properly cited.

(c) Copyright 2019 by the Korean Society of Medical Genetics and Genomics 
condition in $1975[3,4]$. The PIK3R1 gene, found at position 13.1 on the $q$ arm of chromosome 5 ( $5 q 13.1)$, encodes the p85 alpha subunit, whose interaction with insulin receptor substrate-1 is an important part of the phosphoinositide-3-kinase/AKT/ mechanistic target of rapamycin molecular pathway that plays a major role in growth, adipose tissue proliferation, and insulin signaling $[4,5]$. There have been 10 reported pathogenic allelic mutation variants of the PIK3R1 gene that have led to the phenotypic presentation of SHORT syndrome. SHORT syndrome arises spontaneously as a de novo mutation and can be passed on via autosomal dominant inheritance, resulting in families with similar manifestations of the condition [3]. 3q duplication syndrome consists of an expansive list of possible malformations as follows: facial irregularities, limb deformities, heart defects, developmental delay, cerebral and cerebellar malformations, renal dysplasia, and musculoskeletal anomalies [6]. The incidence of $3 q$ duplication syndrome is $<1 / 1,000,000$ with approximately 50 cases being reported in literature [6]. There have been reported cases of varying types of mutations on chromosomes 3 and 5 simultaneously $[7,8]$. To our knowledge there are no documented cases of concurrent $3 q$ duplication syndrome and SHORT syndrome.

\section{Case}

Our patient was born at term via cesarean section to a 26-year-old gravida 2, para 1 mother with no significant past medical history and no family history of genetic syndromes. The mother was not exposed to any teratogenic substances, but pregnancy was complicated by IUGR diagnosed by abdominal ultrasound. The patient was born small for gestational age, weighing 1,956 g. In addition to his small size, on newborn physical examination the patient was noted to have a number of physical aberrations as follows: bossing of frontal bone of skull, triangular shaped face, micrognathia, sunken eyes, cleft palate, thin wrinkled skin (progeroid appearance), undescended left testicle and marked lipodystrophy of the upper and lower limbs (Table 1) [1,9]. All fingers and toes were present and no hand or feet anomalies were noted. No heart murmurs were auscultated and the patient has not received an echocardiogram. The patient's cleft palate caused complications with feeding by interfering with proper latch to the nipple for breastfeeding and required a gastric tube be placed for one month. As the patient grew, feeding normalized. The cleft palate is not large enough to cause eating or breathing problems for the patient and has subsequently not been repaired. Respiratory distress due to pul- monary hypoplasia resultant of IUGR necessitated oxygen supplementation for one-month post-partum. At age 4-months, the patient underwent a screening audiology evaluation that indicated his hearing was within normal limits. A single nucleotide polymorphism (SNP) array done via ExomeNext-Select by Ambry Genetics (Aliso Viejo, CA, USA) at 8 months old showed a pathogenic missense mutation of the PIK3R1 gene on chromosome $5 q 13.1$, an autosomal dominant, heterozygous, de novo mutation diagnostic for SHORT syndrome and the Ambry chromosomal microarray: SNP Microarray (Affymetrix CytoScan HD Array, Santa Clara, CA, USA) showed a pathogenic gain mutation on chromosome $3 q 25$ diagnostic for $3 q$ duplication syndrome. Clinical screening indicated that no one in the patient's family suffers from the same condition. Patient was referred to an ophthalmologist to rule out Rieger anomaly at age 1 year and 5 months and was found to have normally formed anterior and posterior segments of the eyeball as well as normal visual acuity. At age 2 , the patient was in the $<2 \%$ range for both length (81 $\mathrm{cm}$ ) and weight $(9.5 \mathrm{~kg})$. The patient has met social and learning developmental milestones consistently from birth and shows no signs of intellectual disability or repetitive behaviors. Physical

Table 1. Patient manifestations vs. common manifestations of SHORT syndrome and $3 q$ duplication syndrome

\begin{tabular}{|c|c|c|c|}
\hline Manifestation & $\begin{array}{l}\text { SHORT } \\
\text { syndrome }\end{array}$ & $\begin{array}{l}\text { 3q duplication } \\
\text { syndrome }\end{array}$ & Patient \\
\hline Short stature & + & - & + \\
\hline Hyperextensibility of joints & + & - & - \\
\hline Inguinal hernia & + & - & - \\
\hline Ocular depression & + & - & + \\
\hline Rieger anomaly & + & - & - \\
\hline Teething delay & + & + & + \\
\hline Progeroid face & + & - & + \\
\hline Lipodystrophy & + & - & + \\
\hline Intrauterine growth restriction & + & - & + \\
\hline Hearing loss & + & - & - \\
\hline Vision changes & + & + & - \\
\hline Frontal bossing & + & + & + \\
\hline Micrognathia & + & + & + \\
\hline Early feeding difficulty & + & + & + \\
\hline Slow physical development & + & + & + \\
\hline Cardiac defects & - & + & - \\
\hline Cleft palate $^{\mathrm{a}}$ & - & + & + \\
\hline Cryptorchidism ${ }^{b}$ & - & + & + \\
\hline
\end{tabular}

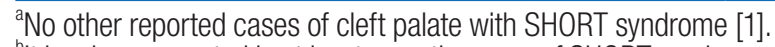

'It has been reported in at least one other case of SHORT syndrome [9]. SHORT, short stature, hyperextensibility of joints and/or inguinal hernia, ocular depression, Rieger anomaly, and teething delay; +, positive; -, negative. 
developmental milestones have been consistently delayed. As of age 2 years and 3 months, the patient is pending echocardiogram results to definitively rule out cardiac abnormalities, he has no evidence of insulin resistance, and is scheduled for surgery to repair his undescended left testicle.

\section{Discussion}

This patient has many of SHORT syndrome's prime manifestations including short stature, delayed teeth eruption, IUGR, progeroid appearance, facial gestalt, lipodystrophy, and ocular depression. He suffers from one of the 10 reported variants of the SHORT syndrome mutation, c.1945C $>$ T, p.R649W, a substitution missense mutation that substitutes a cytosine base for a thymine at nucleotide position 1945 resulting in an arginine being changed to a tryptophan at amino acid position 649. This mutation, along with c.1929_1933delTGGCA, c.1615_1617delAT, c.1465G>A, c.1943dupT, and c.1892G>A have been associated with adolescent onset of insulin resistance [10]. This patient is too young to have developed insulin resistance at this point but will most likely develop this complication by his teenage years. There was one case report of an 18-yearold with c. 1945C > T mutation that did not develop this problem [11]. Lipodystrophy frequently appears with insulin resistance and has been associated with the c. 1954C>T mutation as well and given that our patient has lipodystrophy and the associated mutation, he will most likely develop insulin resistance in the future. Rieger anomaly, which is not present in this case, has been documented in a case series of two out of five patients with c. 1945C>T mutation [11]. Geographically, the condition does not have a consistent distribution with recent cases occurring in India, Poland, and the United States (this patient) $[9,12]$.

The patient was born with both a cleft palate and cryptorchidism, neither of which are currently defined as being aspects of SHORT syndrome. One previous case has reported the co-occurrence of SHORT syndrome and cryptorchidism [9]. Cleft palate and cryptorchidism have both been associated with $3 q$ duplication syndrome, the second pathogenic chromosomal mutation this patient suffers from, leading us to believe that $3 q$ duplication syndrome is the more likely cause of these malformations. $3 q$ duplication syndrome has a wide variation of presentations based on the size of the mutation and the location of the mutation within the $q$ arm of chromosome $3.3 q$ duplication mutations are autosomal dominant gain of function mutations that lead to manifestations based on the size of the mutation and the location within the $q$ arm of chromosome 3 [6]. Our patient has a gain of function mutation approximately 3.39 megabases in size spanning the $3 q 25.31-3 q 25.33$ regions. Two characteristics more frequently seen in $3 q 25$ mutations than at other mutation positions are cleft palate/lip and clubfoot. Our patient was found to have cleft palate but did not have any pedal malformations. Also, he does not have many other aspects of $3 q$ duplication syndrome including: brain malformations, hearing loss, loss of visual acuity, genitourinary malformations, seizures, and cardiac malformations. A compilation of 50 cases of $3 q$ duplication syndrome created by Unique, a rare chromosome disorder support group, states that one out of three patients with a gain of function mutation at 3q25 present with cleft palate [6]. This finding is consistent with our patient's SNP array and manifestations.

The differential diagnosis for SHORT syndrome consists primarily of four disorders: Russell-Silver syndrome, Alagille syndrome, Berardinelli-Seip congenital lipodystrophy, and Hutchinson-Gilford progeria syndrome [2]. Russell-Silver syndrome manifests with overlapping features of SHORT syndrome including IUGR, short stature, and similar facial irregularities such as triangular face but does not present with lipodystrophy, ocular abnormalities, or teething delays [2]. Alagille syndrome is similar to SHORT syndrome in that both present with ocular and dental abnormalities, but Alagille consists of hepatic dysfunction not present in SHORT syndrome [2]. Berardinelli-Seip congenital lipodystrophy overlaps with SHORT syndrome in that they both present with lipodystrophy and insulin resistance. However, the lipodystrophy found in Berardinelli-Seip is much more severe and although facial feature abnormalities are present, they resemble acromegaloid features as opposed to the smaller, triangular-shaped progeroid appearance characteristic of SHORT syndrome [2]. Hepatomegaly is also seen in BerardinelliSeip, a feature that has not been reported in SHORT syndrome. Hutchinson-Gilford progeria syndrome similarly presents with lipodystrophy and the progeroid appearance, but unlike SHORT syndrome, these symptoms do not develop until childhood whereas patients with SHORT syndrome present at birth.

The mnemonic 'SHORT' has become increasingly inaccurate when clinically describing patients with new incidences of the condition. Avila and colleagues [1] retrospectively reviewed 32 patients with SHORT syndrome and concluded that many of the manifestations described in the mnemonic are not present in a majority of affected individuals. Additionally, our patient lacks many of the aforementioned notable features including hyperextensibility of joints, inguinal hernia, Rieger anomaly, hearing loss, and vision loss, findings consistent with the metaanalysis. Of these absent symptoms, hyperextensibility of joints, 
Table 2. SHORT syndrome features

\begin{tabular}{|lc|}
\hline \multicolumn{1}{|c}{ Acronym feature } & Patients \\
\hline Short stature & $26 / 32$ \\
\hline Hyperextensible joints & $10 / 30$ \\
\hline Ocular depression & $28 / 28$ \\
\hline Rieger anomaly & $13 / 31$ \\
\hline Teething delay & $21 / 21$ \\
\hline Facial gestalt & $33 / 33$ \\
\hline Lipodystrophy & $27 / 30$ \\
\hline Intrauterine growth restriction & $23 / 27$ \\
\hline
\end{tabular}

SHORT, short stature, hyperextensibility of joints and/or inguinal hernia, ocular depression, Rieger anomaly, and teething delay.

Revised from the article of Avila M et al. (Clin Genet 2015;89:501-6) [1] with copyright holder's permission.

inguinal hernia, and Rieger anomaly are included in the SHORT mnemonic despite only being clinically present in 33\% (10/30 patients with diagnosed SHORT syndrome tested for anomaly), $33 \%$ (10/30), and 43\% (13/31) of cases respectively [1]. Metaanalysis consists of 32 cases, but not all cases were tested for all specific manifestations. Our patient has been included in these statistics. Contrarily, many of the most common clinically present aspects of SHORT syndrome are excluded from the naming schemes, including facial gestalt (33/33, 100\%), lipodystrophy (27/30, 90\%) and IUGR (23/27, 85\%) (Table 2). The remaining presentations within the SHORT mnemonic are clinically present $81 \%$ to $100 \%$ of the time: short stature $(26 / 32 ; 81 \%)$, ocular depression (28/28; 100\%), and teething delay $(21 / 21 ; 100 \%)$ [1].

\section{Acknowledgements}

We would like to extend a special thank you to the family of the patient, Dr. Beesley's office staff, and Ross University School of Medicine.

\section{References}

1. Avila M, Dyment DA, Sagen JV, St-Onge J, Moog U, Chung BHY, et al. Clinical reappraisal of SHORT syndrome with PIK3R1 mutations: toward recommendation for molecular testing and management. Clin Genet 2015;89:501-6.
2. Innes AM, Dyment DA. SHORT syndrome. In: Adam MP, Ardinger HH, Pagon RA, Wallace SE, eds. GeneReviews ${ }^{\circledR}$ [Internet]. Seattle (WA): University of Washington, 2014 May 15 [cited 2018 Apr 15]. [https:// www.ncbi.nlm.nih.gov/books/NBK201365]

3. The Online Mendelian Inheritance in Man. \# 269880: SHORT syndrome [Internet]. Baltimore (MD): McKusick-Nathans Institute of Genetic Medicine, Johns Hopkins University School of Medicine, 1986 Jun 4 [updated 2017 Dec 14; cited 2018 Apr 15]. [https://www.omim.org/ entry/269880?search=short\%20syndromecthighlight=syndromic $\% 20$ short\%20syndrome\#]

4. Orphanet: SHORT syndrome [Internet]. Paris: INSERM [updated 2015 Dec; cited 2018 Apr 8]. [http://www.orpha.net/consor/cgi-bin/OC_ Exp.php?Lng=GBCExpert=3163]

5. Chudasama KK, Winnay J, Johansson S, Claudi T, König R, Haldorsen I, et al. SHORT syndrome with partial lipodystrophy due to impaired phosphatidylinositol 3 kinase signaling. Am J Hum Genet 2013:93:150-7.

6. Unique (Understanding Rare Chromosome and Gene Disorders). Duplications of 3q. [Internet]. Oxted: Rare Chromosome Disorder Support Group, 2008 [cited 2018 Mar 12]. [https://www.rarechromo.org/ media/information/Chromosome\%20\%203/3q\%20duplications\%20 FTNW.pdf]

7. Angle $B$, Yen $F$, Hersh JH, Gowans G. Patient with terminal duplication $3 q$ and terminal deletion $5 q$ : comparison with the $3 q$ duplication syndrome and distal $5 q$ deletion syndrome. Am J Med Genet A 2003;116A:376-80.

8. Preiksaitiene $E$, Benušienė $E_{1}$ Ciuladaite $Z$, Šliužas V, Mikštienė V, Kučinskas $V$. Recurrent fetal syndromic spina bifida associated with 3q26.1-qter duplication and 5p13.33-pter deletion due to familial balanced rearrangement. Taiwan J Obstet Gyneco 2016;55:410-4.

9. Singh $A$, Arora $R$, Singh $P_{1}$ Kapoor $S$. Short syndrome-an expanding phenotype. Indian Pediatr 2013;50:414-6.

10. Thauvin-Robinet $C_{1}$ Auclair M, Duplomb L, Caron-Debarle $M$, Avila $M$, St-Onge J, et al. PIK3R1 mutations cause syndromic insulin resistance with lipoatrophy. Am J Hum Genet 2013;93:141-9.

11. Dyment DA, Smith AC, Alcantara D, Schwartzentruber JA, Basel-Vanagaite $L_{1}$ Curry $C J$, et al. Mutations in PIK3R1 cause SHORT syndrome. Am J Hum Genet 2013;93:158-66.

12. Klatka M, Rysz I, Kozyra K, Polak A, Kołłątaj W. SHORT syndrome in a two-year-old girl-case report. Ital J Pediatr 2017;43:44. 